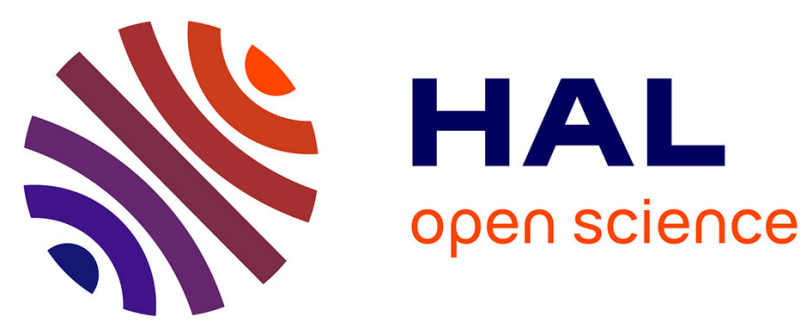

\title{
Investigation of aluminum droplet combustion in solid propellant flames: Al-PLIF experiments and numerical simulation
}

Pier-Henri Chevalier, Nelly Dorval, Robin Devillers, Dmitry Davidenko, Xavier Mercier, Julien Pichillou

\section{To cite this version:}

Pier-Henri Chevalier, Nelly Dorval, Robin Devillers, Dmitry Davidenko, Xavier Mercier, et al.. Investigation of aluminum droplet combustion in solid propellant flames: Al-PLIF experiments and numerical simulation. AIAA Propulsion and Energy 2021 Forum, Aug 2021, Virtual event, United States. pp.AIAA 2021-3618, 10.2514/6.2021-3618 . hal-03440174

\author{
HAL Id: hal-03440174 \\ https://hal.science/hal-03440174
}

Submitted on 22 Nov 2021

HAL is a multi-disciplinary open access archive for the deposit and dissemination of scientific research documents, whether they are published or not. The documents may come from teaching and research institutions in France or abroad, or from public or private research centers.
L'archive ouverte pluridisciplinaire HAL, est destinée au dépôt et à la diffusion de documents scientifiques de niveau recherche, publiés ou non, émanant des établissements d'enseignement et de recherche français ou étrangers, des laboratoires publics ou privés. 


\title{
Investigation of aluminum droplet combustion in solid propellant flames: Al- PLIF experiments and numerical simulation
}

\author{
P-H. Chevalier ${ }^{1}$, N. Dorval ${ }^{2}$, R. Devillers ${ }^{3}$ and D. Davidenko ${ }^{4}$ \\ ONERA, Paris-Saclay University, F-91123 Palaiseau, France
}

X. Mercier $^{5}$

Lille University, CNRS, UMR 8522 - PC2A Lab, F-59000 Lille, France

J. Pichillou 6

CNES, the Launcher Directorate, 75612, Paris, France

\begin{abstract}
Aluminum-droplet combustion is studied in solid-propellant flame environment at 1.0 MPa. Al vapor is observed during the combustion process with high-speed Al-PLIF and visible-emission images. A onedimensional quasi-steady model of aluminum combustion is used to simulate Al-atom concentration and temperature profiles around the droplet. AI-PLIF image simulation has been developed to generate synthetic fluorescence images that reproduce the experimental images. A comparison approach is initiated between experimental and numerical Al-PLIF images and applied to the study of a specific droplet observed during the experiments with a diameter larger than $120 \mu \mathrm{m}$. A reasonable consistency is observed between simulated and experimental data, which is promising to provide validation data for the development of more representative aluminum-combustion models.
\end{abstract}

\section{Nomenclature}

\section{Combustion}

$D_{p} \quad=$ droplet diameter

$R_{p} \quad=$ droplet radius

$R_{e} \quad=$ external border simulation in polar coordinate

$T=$ gas temperature

$T_{a} \quad=$ equilibrium ambient gas temperature

$[A l]_{p}=$ Surface droplet Al concentration

$[\mathrm{Al}]=\mathrm{Al}$ concentration

$P \quad=$ static pressure

$N_{A l} \quad=$ total number density of $\mathrm{Al}$ atoms

$N_{A l, i}=$ number density of probed atoms in the initial electronic state $\mathrm{i}$

\footnotetext{
${ }^{1}$ PhD Student, DPHY/SLM, email: pier-henri.chevalier@onera.fr

${ }^{2}$ Research Scientist, DPHY/SLM

${ }^{3}$ Research Scientist, DMPE/MPF

${ }^{4}$ Research Scientist, DMPE/MPF

${ }^{5}$ Director, Laboratory PC2A

${ }^{6}$ Solid Propellant Devices Expert, CNES/DLA
} 


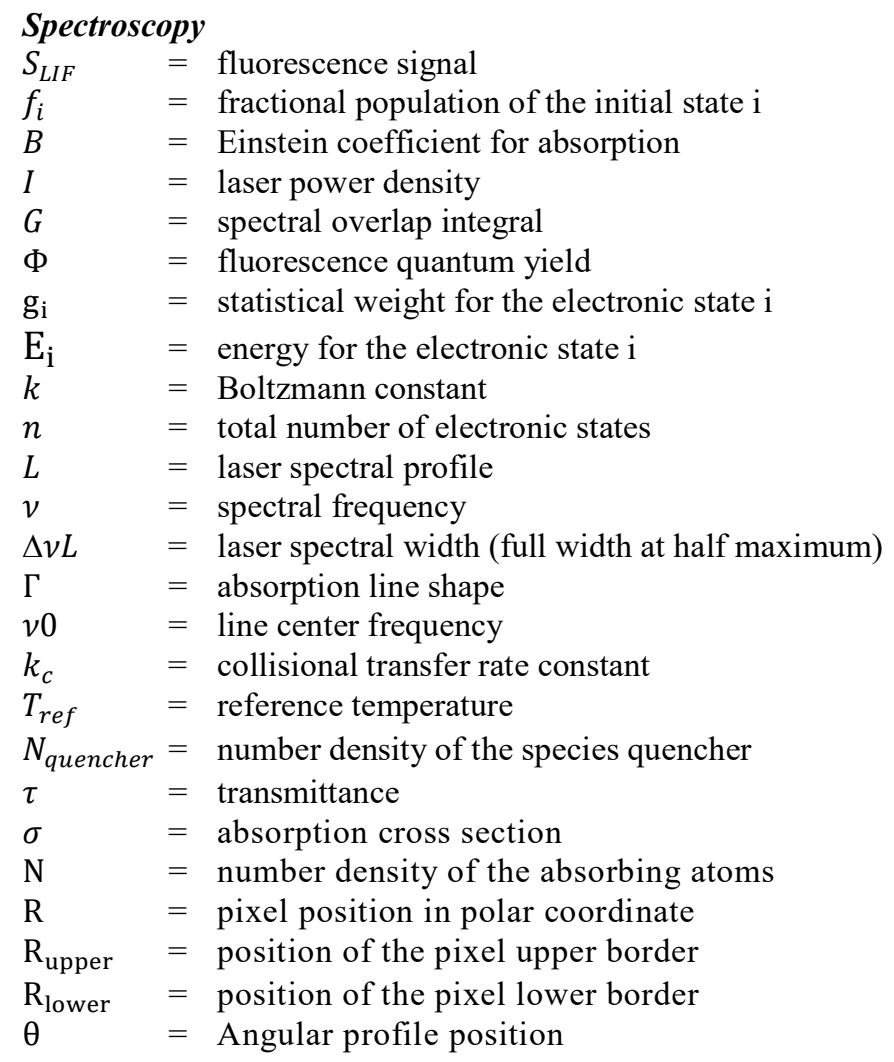

\section{Introduction}

Solid propulsion is widely used for space and military applications. Solid-propellant compositions are commonly based on ammonium perchlorate (AP) particles mixed within a hydroxyl-terminated polybutadiene (HTPB) binder. Aluminum is used to enhance rocket ballistic performances. Its combustion increases adiabatic flame temperature in the combustion chamber of the solid propulsion motor (SPM) and improves specific impulse. During the aluminumdroplet combustion an oxide cap is formed and carried out in the solid-propellant gas flows. The largest aluminum droplets might interact with longitudinal modes due to SPM geometry, which has a negative impact on combustion stability and might be the cause of pressure oscillations [1][2]. The phenomena take place close to the solidpropellant burning surface and define the combustion zone. Its thickness is characteristic of the droplets combustion time from their detachment from the solid-propellant surface to their complete combustion [3]. The estimation of the combustion thickness requires accurate Al-combustion simulation [4] with specific kinetic models [5][6]. The development of models requires accurate experimental data to be confronted to. However, the combustion chamber environment is complex and highly reactive with high temperature and pressure levels. Few data are then available from the literature in such conditions. Most of the time, the environment is reproduced in academic facilities using a stream of monodisperse, freely falling and burning droplets using laser ignition technique [7][8], levitating droplets [9] or solid-propellant flames from small samples [10][11]. The particles burning time is the main studied data in most studies, which is limited for fine model adjustments. Concentration measurements for specific species are sparse [8] but necessary for the validation and improvement of kinetic combustion models. Literature is not extensive for quantitative experimental data for representative aluminum-droplet combustion. For instance, spatial profiles of flame temperature and $\mathrm{AlO}$ concentration measured by planar laser-induced fluorescence technique (PLIF) at low pressure in well-controlled gaseous atmosphere from the Bucher's works are still used for comparison with models developed by several teams [12][13]. PLIF imaging at high-repetition rate $(5 \mathrm{kHz})$ of $\mathrm{Al}$ atom (AlPLIF) has been recently applied in solid-propellant flames at $1.5 \mathrm{MPa}$ [11]. To our knowledge, this was the first time that LIF imaging technique was applied to aluminized propellant flames. A good signal-to-noise ratio due to strong Al-atom signal strength has improved the LIF sensitivity in solid-propellant flames at high pressure. In the present 
work, direct visualization of burning droplets has been coupled to AL-PLIF imaging for droplet size and morphology analysis.

The goal of this paper is to present a method that enables a comparison between measured and calculated Al-PLIF fields surrounding the burning droplet obtained under representative solid-propellant flame conditions. The first goal of this study is to elaborate a process for reconstruction of LIF-signal maps for Al atoms. The task involves two models, one for Al-atom LIF signal and one for aluminum-droplet combustion. The second goal is to compare the calculated Al-PLIF images with the measured ones. Various parameters have been adjusted such as the position of the laser axis with respect to the droplet axis, the radial position of the outer boundary close to droplet to simulate the effect of the ambient gas convection. Comparison between the experimental and calculated radial profiles of Al LIF signal is presented.

\section{Experimental apparatus}

Planar laser-induced fluorescence on aluminum atoms in gas phase (Al-PLIF) is used to study aluminum droplets combustion taking place above the surface of burning solid-propellant samples (with pressure up to $1.5 \mathrm{MPa}$ ). Al atoms produce a significant fluorescence signal that can be easily detected even with the harsh conditions of the solid-propellant flames (high-luminosity, high-pressure and heavily particle-laden environment). In our laboratory, metallic atoms such as iron and aluminum have been probed during solid-propellant combustion [11][14]. Al-PLIF at high repetition-rate $(5 \mathrm{kHz})$ was shown to be quite informative about the Al-droplet combustion process close to the propellant surface and above, during the short combustion duration ( $\sim \mathrm{s})$ of aluminized propellant samples [14]. In the present work, simultaneous Al-vapor fluorescence and droplet emission images are recorded in order to improve analysis of $\mathrm{Al}$ droplets behaviour.

\section{A. PLIF and visible-emission imaging setup}

High-resolution imaging of burning aluminium droplets was performed in a combustion chamber designed for optical diagnostics of solid-propellant flames up to a pressure of $1.5 \mathrm{MPa}$. The solid-propellant combustion chamber and the Al-PLIF setup were previously presented [14][15]. The spectroscopic background of the Al atom was described in details elsewhere together with the choice for the excitation and detection scheme [16]. The layout of the coupled Al-PLIF and visible emission imaging is depicted in Figure 1.

High repetition rate and short laser pulses (nanosecond) are needed for acquiring time-resolved images of these flames with short combustion duration $(\sim 1 \mathrm{~s})$ and high level of emission background. We used a high-speed LIF system that is composed of a tunable narrow-linewidth dye laser (Credo, Sirah Lasertechnik) pumped by a solidstate laser (INNOSLAB IS2011-E, EdgeWave) operating at $532 \mathrm{~nm}$ and $5 \mathrm{kHz}$ repetition rate. The dye laser operates with a mixture of Rhodamine dyes (Rh610 and Rh640). The resulting beam at $618 \mathrm{~nm}$ is then frequencydoubled using a BBO crystal. The UV laser wavelength is tuned to the ${ }^{2} \mathrm{P}_{3 / 2}^{\circ}-{ }^{2} \mathrm{D}_{5 / 2}$ transition of $\mathrm{Al}(309.361 \mathrm{~nm}$, vacuum wavelength). The laser beam is focussed into a thin sheet using a system of spherical and cylindrical lenses (sheet height of $17 \mathrm{~mm}$ and thickness of $150 \mu \mathrm{m}$ ). The laser energy is measured to be $50 \mu \mathrm{J}$ per pulse at the chamber entrance. The pulse duration is $5 \mathrm{~ns}$. The distribution of laser intensity over the height of the laser sheet is measured from liquid acetone fluorescence images in order to correct the spatial non-uniform laser excitation as well as to account for shot-to-shot fluctuations of the laser energy (in the range of $\pm 15 \%$ ).

For the present experimental investigation, two high-speed cameras in combination with two long-distance microscope objectives (QM-100, Questar) are mounted at $90^{\circ}$ in order to record simultaneously both gaseous Al atoms fluorescence and visible emission from the burning droplet. A dichroic mirror (425 nm cut-off wavelength) reflecting the UV fluorescence light from Al atoms and transmitting the visible radiation is used (Fig. 1). The cameras are set on translation stages that enable fine spatial adjustments in order to match the objective focal planes onto the laser plane as well as the cameras fields of view with respect to the sample.

For Al-atom fluorescence detection, a 12-bit CMOS camera (HSS6, LaVision) is used with a high-speed intensifier (HS-IRO, LaVision). Fluorescence light at $394 \mathrm{~nm}$ and $396 \mathrm{~nm}$ corresponding respectively to the ${ }^{2} \mathrm{P}_{1 / 2}-$ ${ }^{2} \mathrm{~S}_{5 / 2}$ and ${ }^{2} \mathrm{P}_{3 / 2}^{\circ}-{ }^{2} \mathrm{~S}_{5 / 2}$ transitions of $\mathrm{Al}$ is selected by using a bandpass filter centred at $394 \mathrm{~nm}(\mathrm{FWHM}=10 \mathrm{~nm})$ [16]. Rejection of the scattered light is enhanced by adding a long-pass filter ( $385 \mathrm{~nm}$ cut-on wavelength). For the visible emission imaging, a 12-bit CMOS Phantom V711 camera is used. A neutral-density filter is added to attenuate the visible radiation.

The frame rate and the image resolution are set, respectively, to $10 \mathrm{kHz}$ and $768 \times 768$ pixel for both cameras. For LIF imaging, the frame rate is twice larger than the laser repetition rate enabling alternate acquisition of LIF and 
UV emission images. The intensifier gate width is set to $40 \mathrm{~ns}$ in order to obtain a good signal-to-background ratio. We have checked temporal jitter of the laser/intensifier gate system does not affect the signal intensity. Furthermore, the elastic light scattering contribution due to Al droplets and alumina smoke was measured to be negligible by recording images when the laser wavelength was tuned off resonance in a dedicated combustion test. Accordingly, the recorded signal arises mainly from Al vapor fluorescence. The intensifier gain was adjusted in order to avoid local saturation of the CMOS sensor. The exposure time of the visible camera is set to $4 \mu \mathrm{s}$. The fields of view ( $4 \times$ $4 \mathrm{~mm}^{2}$ ) are made identical by carefully aligning the two cameras using a resolution test target. Thanks to a careful alignment protocol, it was possible to match the depths of field for both microscope objectives (approximately 100$\mu \mathrm{m}$ thick) to the laser-sheet thickness of $150 \mu \mathrm{m}$. Laser-excitation pulses and the two cameras are temporally synchronized using the DaVis software. The cameras are synchronized with an external trigger signal delivered by the $\mathrm{CO}_{2}$ laser used for ignition, which gives a reference time for the recording images. A large number of images $(\sim$ 20,000) are recorded during each combustion test.

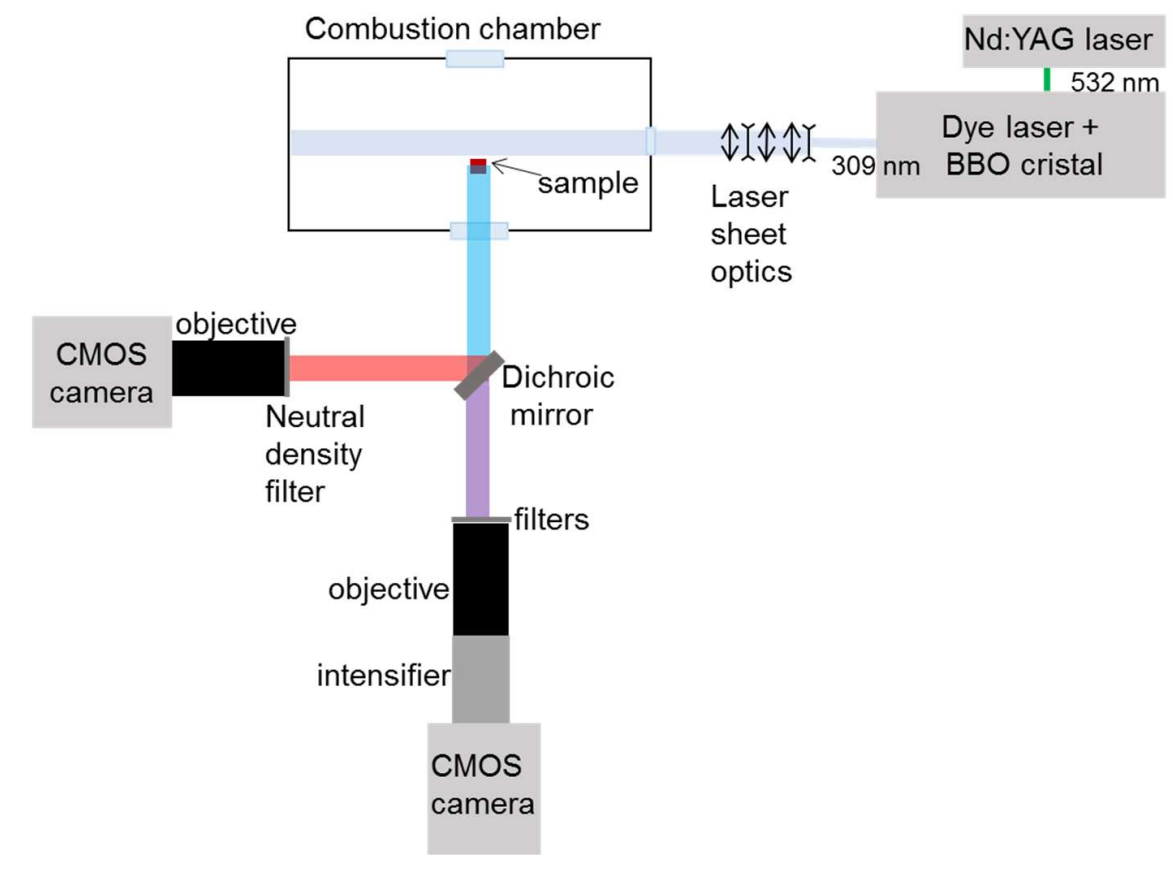

Figure 1. Optical set-up used for PLIF and visible emission imaging experiments.

\section{B. Propellant combustion conditions}

The combustion chamber was described previously [11]. The chamber is pressurized with nitrogen at pressure from 1.0 to $1.5 \mathrm{MPa}$ in the present experiment. Small solid-propellant samples $(4 \times 5 \times 6 \mathrm{~mm})$ are maintained in a holder placed at the center of the chamber. $\mathrm{A} \mathrm{CO}_{2}$ laser beam enters from the top of the chamber through a $\mathrm{ZnSe}$ window in order to ignite the sample. Pulse energy and duration were adjusted in order to reach ignition between 50 and $100 \mathrm{~ms}$. The holder is kept fixed during the burning time so that the surface regression is not compensated.

The aluminized solid-propellant composition burnt during the campaign is made of ammonium perchlorate (AP) and aluminum particles embedded into a hydroxyl-terminated polybutadiene (HTPB) binder. The present composition has an aluminum mass fraction of $18 \%$, which is common in solid-propellant applications. Diameter for the individual aluminum particle used to produce the solid-propellant sample ranges from 10 to $120 \mu \mathrm{m}$ but droplets with larger diameter are observed above the burning solid-propellant surface because of agglomeration processes.

\section{Images post-processing and observations}


Various processing steps are performed in order to select droplets with significant fluorescence signal level and follow it over time. The first step aims at taking into account the laser-fluence inhomogeneity over the vertical axis in the laser sheet. Acetone-PLIF is measured by placing a small liquid-acetone in the chamber before the combustion test in order to evaluate the vertical laser-fluence profile. Al-PLIF images are divided by the subsequent laser-fluence vertical profile to compensate for the inhomogeneous laser fluence. Over the image height, the laserfluence inhomogeneity remains small with a standard deviation below $10 \%$. The laser-fluence standard deviation remains smaller than $4 \%$ over the typical size of $\mathrm{Al}$ droplets.

The second processing aspect is the image registration for the two cameras e.g., fluorescence images and visible-emission images. Image registration is obtained from images of a spatial reference grid, acquired with both cameras in-between combustion tests. It enables correcting for discrepancy in terms of spatial resolution as well as evaluating the required translation so that the two images match spatially. Image registration is a crucial step in order to associate each detected droplet showing fluorescence signal to the proper droplet from the visible-image, which is used to estimate the droplet diameter. The accuracy for the automatic grid-based image registration is not perfect though and manual adjustments were also involved to estimate the droplet position relative to the detected fluorescence image.

Image analysis also includes detecting automatically the droplets with significant fluorescence signal level, and track them over various images. The detection step is performed with the Maximally-Stable Extremal Region algorithm [19] whose efficiency was demonstrated previously for Al-PLIF images [15]. The algorithm is applied only to the fluorescence images (with laser-excitation on) in order to detect the droplet with fluorescence signal. A Kalman filter is applied to analyze the detected fluorescent regions over various images so that the same droplet is tracked over time. This way, droplet velocity is evaluated and the Al fluorescence signal evolution is followed over time. The association of detected UV-signal regions for successive images is labelled "track" in the article.

The last analysis step corresponds to droplet-diameter evaluation for the tracks of interest. It corresponds to the analysis of the visible images using gradient levels. Both droplet and flame diameters are estimated by drawing circles manually on the gradient image. Gradient images are only annotated for droplets of interest over their successive positions. Annotation gives access to droplet-diameter estimation as well as droplet velocity values. Diameter is a required input parameter for any Al-combustion simulation. Droplet and gas velocity are not used in combustion simulations for the present study since only 1D simulations were performed, as described in section IV.A, but they will be necessary for future 2D simulations.

\section{IV.Al-PLIF image simulation}

In this section, we describe our theoretical approach to simulate Al fluorescence images in solid-propellant flame conditions, which is required to validate models of aluminum-droplet combustion and improve the understanding of recorded fluorescence images. Reconstruction of maps for Al-vapor fluorescence signal relies on two models, one for Al-atom LIF signal and one for aluminum-droplet combustion. A simulated Al-PLIF image can be generated by using the method described below in subsection B. This method requires input data on the Al-atom concentration and temperature within the laser sheet. In the present study, such data are obtained by simulating combustion of an aluminum droplet in a 1D approach outlined in subsection A. The proposed simulation method will be used to generate synthetic fluorescence images that reproduce the observed images in a real combustion sequence.

\section{A. Aluminum-droplet combustion modelling}

Aluminum droplet combustion is modeled using a one-dimensional (1D) quasi-steady-state approach. This 1D model is described in detail in [20][21]. Here the modeling approach is briefly outlined to give its main features.

The aluminum droplet is considered as a sphere of diameter $D_{p}$. With the quasi-steady-state approach, the droplet diameter and chemical composition are fixed and its thermal state is adiabatic. Mass exchange between the bulk material, which constitutes the droplet, and the surrounding gas is controlled by surface reactions including Al evaporation. All the surface species are at equilibrium with zero net production rates.

An alumina cap is taken into consideration as a spherical segment of diameter $D_{p}$. Its external surface is supposed to be chemically inert, hence the cap overall effect consists in partial inhibition of the aluminum reactive surface.

The reactive gas flow is treated as spherically symmetric, laminar and isobaric (low-Mach approach). The flowfield is governed by a system of transport equations for the energy and chemical species. Molecular transport is 
accurately described with the full multispecies formalism [22]. The rates of chemical species production are determined from a detailed kinetic mechanism.

The gas-flow equations are discretized according to the finite volume method. The convective fluxes are approximated by a hybrid scheme, whose order varies between 1 and 2 depending on the local Peclet number. A second order accurate scheme is used for the molecular fluxes. The computational grid is adapted to minimize the numerical errors by verifying gradient and curvature criteria related to the solution variables.

The computational domain for the gas flow covers a finite radial distance from the droplet surface to the outer boundary. On the outer boundary, the ambient conditions are imposed in terms of temperature and chemical composition. This boundary can be set at an arbitrarily large distance to model droplet combustion in a still environment. By setting the outer boundary close to the droplet, it is possible to model the effect of ambient gas convection. Previously performed computational tests [20][21] show a fairly good agreement between a 2D flowfield with ambient gas convection and a 1D solution with adjusted position of the outer boundary.

\section{B. Al-PLIF image simulation}

\section{$\underline{\text { LIF-signal calculation }}$}

Assuming negligible saturation of the laser excited transition, the fluorescence signal, $S_{L I F}$ is given by [23]:

$$
S_{L I F} \propto N_{A l} f_{i}(T) B I G(T, P) \Phi(T, P)
$$

where $N_{A l}$ is the total number density of atoms excited from the initial electronic state $\left({ }^{2} \mathrm{P}^{\circ} 3 / 2\right)$ to the upper electronic state $\left({ }^{2} \mathrm{D}_{5 / 2}\right)$ by absorption of laser radiation, $f_{i}$ is the fractional population of the initial state function of temperature, B is the Einstein coefficient for absorption, I is the power density of laser, G is the spectral overlap integral and $\Phi$ is the fluorescence quantum yield. The last two terms are function of both temperature and pressure.

At thermal equilibrium, the fractional population is given by the Boltzmann relation:

$$
f_{i}(T)=\frac{g_{i} e^{-\frac{E_{i}}{k T}}}{\sum_{j=1}^{n} g_{j} e^{-\frac{E_{j}}{k T}}}
$$

where $\mathrm{g}_{\mathrm{i}}, \mathrm{g}_{\mathrm{j}}$ are statistical weights, $\mathrm{E}_{\mathrm{i}}, \mathrm{E}_{\mathrm{j}}$ are energies for the electronic states and $\mathrm{k}$ is the Boltzmann constant.

The spectral overlap integral, which contributes to laser excitation efficiency, can be estimated by:

$$
\left.G=\int L(v, \Delta v L) \Gamma(v, v 0, P, T)\right) d v
$$

where $\mathrm{L}$ is the spectral profile of the laser, $\Delta v \mathrm{~L}$ the laser spectral width (full width at half maximum), $\Gamma$ is the absorption line shape function of temperature and pressure, $v 0$ the line center frequency, and integrals of $\mathrm{L}$ and $\Gamma$ over all frequency are normalized to unity. Voigt function is considered for both laser and absorption lines to compute the convolution of line shapes. In combustion media, the broadening of the absorption line shape is mainly governed by collisional and Doppler effects described in general case by a Lorentzian and a Gaussian function, respectively [23].

We have developed a five-level atom model of excitation dynamics to solve the rate equations for each electronic state. This model follows the classical approach used for describing LIF process of atoms but is here complicated by involving multiple levels [23]. The temporal evolution of the number density of Al atoms was calculated in the five levels involved in the selected energy-level scheme of excitation, fluorescence and collisional energy transfers. This theoretical model is described in details in [16][24].

The fluorescence signal is proportional to atom number density but is often complicated by collisional relaxation of the laser excited atoms (collisional quenching). Accordingly, the ratio of fluorescence rate to collisional quenching rate or fluorescence yield $(\Phi)$ is heavily reduced. The collisional dynamics were taken into account in our model through the quenching rates $Q$ for each collisional energy transfers and for the total quenching rate of the 
excited state in the impact approximation. The collisional rate is dependent on pressure, temperature and the nature of the species quenchers and it can be expressed by [23][25]:

$$
\mathrm{Q}(T, P)=k_{c}\left(T_{\text {ref }}\right)\left(\frac{T}{T_{\text {ref }}}\right)^{0.5} N_{\text {quencher }}(T, P)
$$

where $T_{r e f}$ is a reference temperature $(\mathrm{K}), k_{c}\left(\mathrm{~T}_{\text {ref }}\right)$ is the collisional transfer rate constant at $T_{r e f}$ and $N_{\text {quencher }}$ is the number density of the species quencher. Experiments were carried out in thermal evaporation systems of pure aluminum under nitrogen flow in order to determine the total quenching rate for pressures ranging from 1 to $1000 \mathrm{mbar}$ and at high temperature $(1200 \mathrm{~K}$ and $2500 \mathrm{~K})$ [16][24]. As an indicative order of magnitude, a fluorescence quantum yield of $2 \%$ at $0.1 \mathrm{MPa}\left(\mathrm{N}_{2}\right)$ and $2500 \mathrm{~K}$ was estimated and then extrapolated to $0.15 \%$ at $1.5 \mathrm{MPa}$.

In our solid-propellant combustion experiments under high nitrogen pressure (1.0 MPa), we can assume that the main collisional partners are $\mathrm{N}_{2}$ molecules with a constant number density. This assumption allows us to neglect the pressure-dependent issues in fluorescence signal, especially for unknown collisional parameters necessary to account for the other species quenchers encountered in solid-propellant flames. Consequently, the fluorescence signal is assumed to depend only on temperature and on the atom number density. The temperature dependence of the fluorescence signal has been calculated for a temperature range representative of aluminum-droplet combustion ( 2300 to $4000 \mathrm{~K})$. Figure 2 presents how the temperature affects the various terms needed for the $S_{L I F}$ calculation (excluding parameters $\mathrm{B}$ and $\mathrm{I}$ that are independent of $\mathrm{T}$ ).

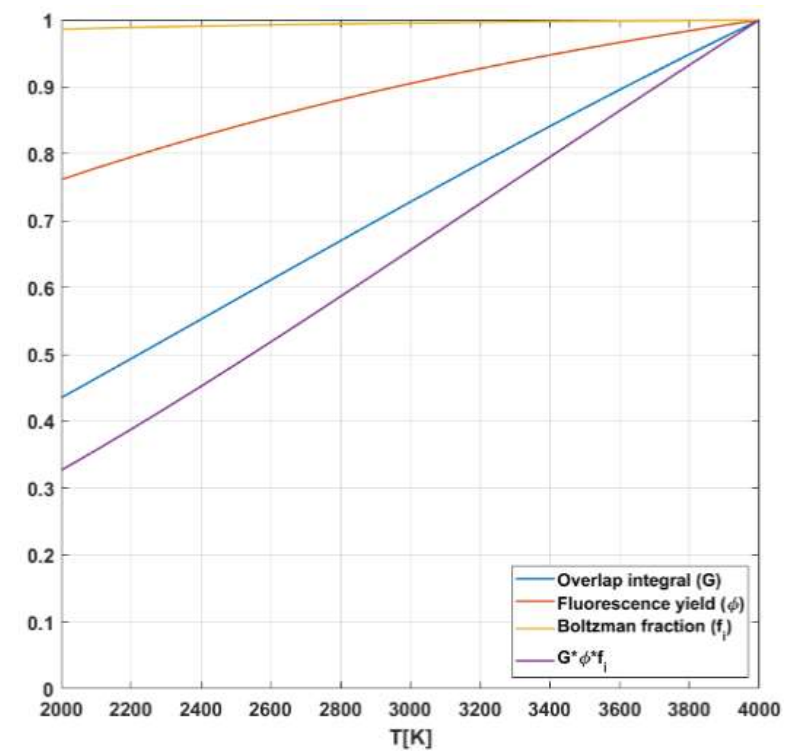

Figure 2. Calculated temperature dependence of each contributing to fluorescence intensity. All parameters normalized to their value at $4000 \mathrm{~K}$.

While the fractional population of the probed state remains nearly constant over the temperature range, the fluorescence yield $(\Phi)$ that is related to the Q rate dependent on $\mathrm{T}$ (Eq. 4) increases by $23 \%$, and the overlap integral $G$ increases by $55 \%$. The effect of $\mathrm{T}$ on the quenching rate and on the absorption lineshape is the most important factor on the temperature sensitivity of the signal. It is worth noting that, although significant, a signal variation of $76 \%$ over this temperature range is not preponderant compared to the variation of $\mathrm{N}_{\mathrm{Al}}$ by several orders of magnitude that is expected around aluminum burning droplets. In any cases, both temperature and concentrations effects are taken into account here for Al-PLIF simulation. In the vapor plume surrounding the burning droplet, the large $\mathrm{Al}$ concentration variation is the major factor for variations in fluorescence signal. 
In counterpart, it is well known that a very large number density of atoms will most likely cause signal losses due to laser energy absorption and fluorescence trapping. A procedure is implemented in the present LIF model to account for both attenuation effects. Transmission has to be evaluated locally because of the gradient levels for concentration and temperature. When considering LIF signal from a spot at location $\mathrm{x}$, the attenuation effect is then obtained by spatial integration of local transmittance along the considered path. Each type of transmittance $\tau$ (laser attenuation, fluorescence trapping) is evaluated by the following formula [26]:

$$
\tau(R)=\exp \left[-\int_{R_{\text {lower }}}^{R_{\text {upper }}} \sigma(x) N(x) d x\right]
$$

where $\sigma$ is the absorption cross section and $N$ the number density of the absorbing atoms. The laser-fluence attenuation for location $\mathrm{R}$ is evaluated by integrating the local transmittance by $\mathrm{Al}$ atoms along the laser path, i.e. from the domain border to location $\mathrm{x}$. The fluorescence-signal trapping is evaluated by integrating local transmittance from the spot in location $\mathrm{x}$ to the camera position. We have calculated the absorption cross-sections corresponding to the laser-excited line $(309 \mathrm{~nm})$ for laser absorption and corresponding to the lines at 394 and $396 \mathrm{~nm}$ for fluorescence trapping, by using spectroscopic data given in [27]. The absorption cross sections are calculated at $1.0 \mathrm{MPa}$ and $3000 \mathrm{~K}$ and are equal to $7.15 \times 10^{-14} \mathrm{~cm}^{2}\left({ }^{2} \mathrm{P}_{3 / 2}-{ }^{2} \mathrm{D}_{5 / 2}\right.$ transition at $\left.309 \mathrm{~nm}\right)$ and to $5.90 \times 10^{-14} \mathrm{~cm}^{2}\left({ }^{2} \mathrm{P}_{1 / 2}^{\circ}-{ }^{2} \mathrm{~S}_{5 / 2}\right.$ and ${ }^{2} \mathrm{P}_{3 / 2}^{\circ}-{ }^{2} \mathrm{~S}_{5 / 2}$ at 394 and $396 \mathrm{~nm}$, respectively).

\section{$\underline{\text { 2D-image calculation }}$}

A simulated Al-PLIF image is obtained by considering fluorescence for all $\mathrm{Al}$ atoms that might interact with the laser sheet and taking into account the local temperature and Al-concentration levels. In practice, temperature and concentration data provided by the combustion simulation are attributed to cells within a 3D domain surrounding the considered Al droplet, as shown in Figure 3. The laser sheet enters the domain from the right-hand side and propagates along the $\mathrm{X}$ axis. The camera is positioned along the $\mathrm{Z}$ axis, perpendicular to the laser sheet. The $\mathrm{Y}$ axis corresponds to the vertical direction along which the hot gases flow during combustion tests. Laser propagation and attenuation along the $\mathrm{X}$ axis and fluorescence trapping along the $\mathrm{Z}$ axis show the need for a 3D domain for Al-PLIF image simulation. In the present study, cells from the calculation domain were chosen with a 1- $\mu \mathrm{m}$ size in order to offer a reasonable spatial resolution for the involved non-linear phenomena (laser attenuation, fluorescence trapping) and the steep gradients.

The Al-combustion simulations are not performed in 3D. Local temperature and Al-concentration values are attributed to each cell by considering symmetry. In the present case, a spherical symmetry is used to generate the local conditions from the 1D temperature and concentration profiles. Combustion simulations for the considered droplet with radius $\mathrm{Rp}$ were performed for domain frontier between 2.0 to $3.0 \mathrm{Rp}$. All Al-PLIF image simulations were computed for 3D domains with cubic 3D domains with side $3.0 \mathrm{Rp}$.

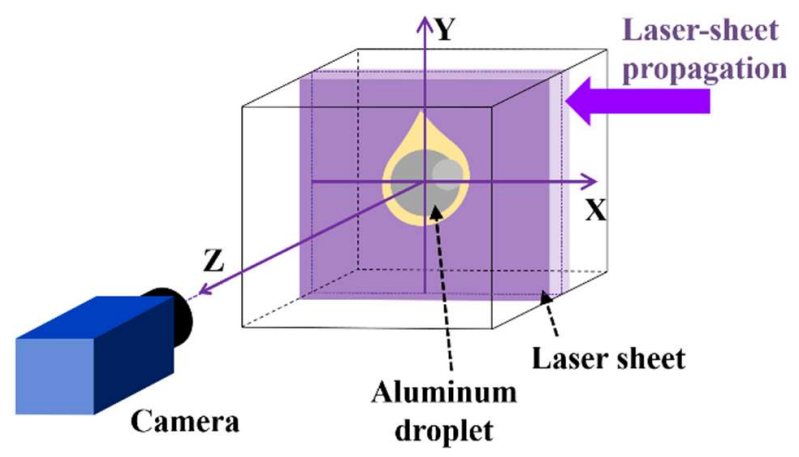

Figure 3. Geometry of the domain for Al-PLIF image simulation. 
The evolution of laser fluence along the laser path accounts for local absorption by Al atoms, estimated for each cell of the domain. The resulting simulated Al-PLIF images are calculated by integrating the emitted fluorescence over the laser thickness, perpendicular to the UV camera and estimating the fluorescence attenuation by trapping along the path to the camera. Similarly to laser attenuation, fluorescence-trapping estimation is accounted for by computing local absorption effects by Al atoms. Optical effects of the imaging objectives were not considered here in terms of depth of field or Point Spread Function but could be added to the image formation step in the future. As will be shown later in the article, the laser-sheet position relative to the Al-droplet has a strong impact on the resulting Al-PLIF image. For each combustion-simulation case, Al-PLIF image were simulated for various lasersheet positions along the $\mathrm{Z}$ axis. As shown in Figure 4, negative position $\mathrm{Z}$ values correspond to a laser-sheet located behind the Al droplet compared to the camera.

a)

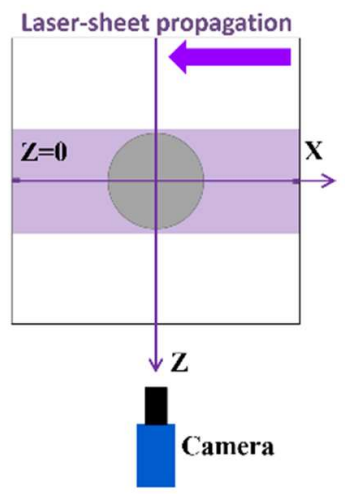

b)

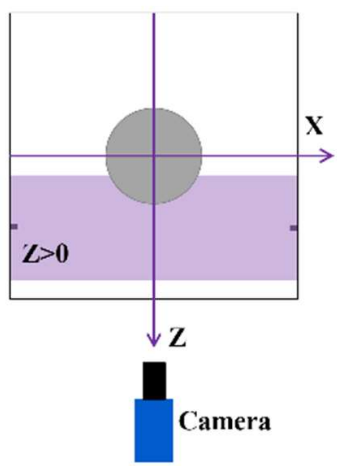

c)

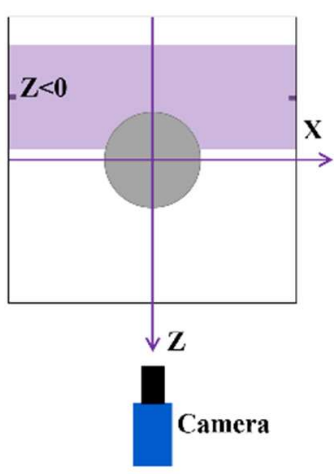

Figure 4. Geometry of the domain for Al-PLIF image simulation for three laser-sheet positions relative to the Al droplet. Top view.

\section{V.Results}

\section{A. Droplet selection}

A droplet was selected with the automatic detection/tracking approach presented previously. The three last tracked positions are shown in Figure 5. The first row shows Al-PLIF images. The spatial distribution and signal intensity change over time. The second row shows UV emission images (i.e. without laser excitation on), recorded after each Al-PLIF images. Intensity signal in the UV emission images is eight times lower than for Al-PLIF images, which confirms that experimental parameters enables analysis of Al-PLIF signal only. The last row corresponds to the visible-emission images taken simultaneously with the Al-PLIF images. The automatic tracking enables to observe the evolution of the morphology, oxide cap position and velocity over time on the successive visible images. The green line corresponds to the trajectory estimated by the tracking algorithm from the automatically detected Al-PLIF areas. Each cross represents the center of the detected LIF signal area. The horizontal position variation of the tracked trajectory is due to size and morphology variations for the detected area. The fluorescence signal shape changes from an oval shape to a quarter-moon crescent shape over the three-image span. The evolution for the fluorescence signal shape could be explained by variations in the laser-sheet relative distance to the droplet. This would be caused by the droplet horizontal displacement relative to the laser sheet. Droplet diameter and position relative to the solid propellant surface are used to simulate the flame characteristics presented in the next sub-section. 


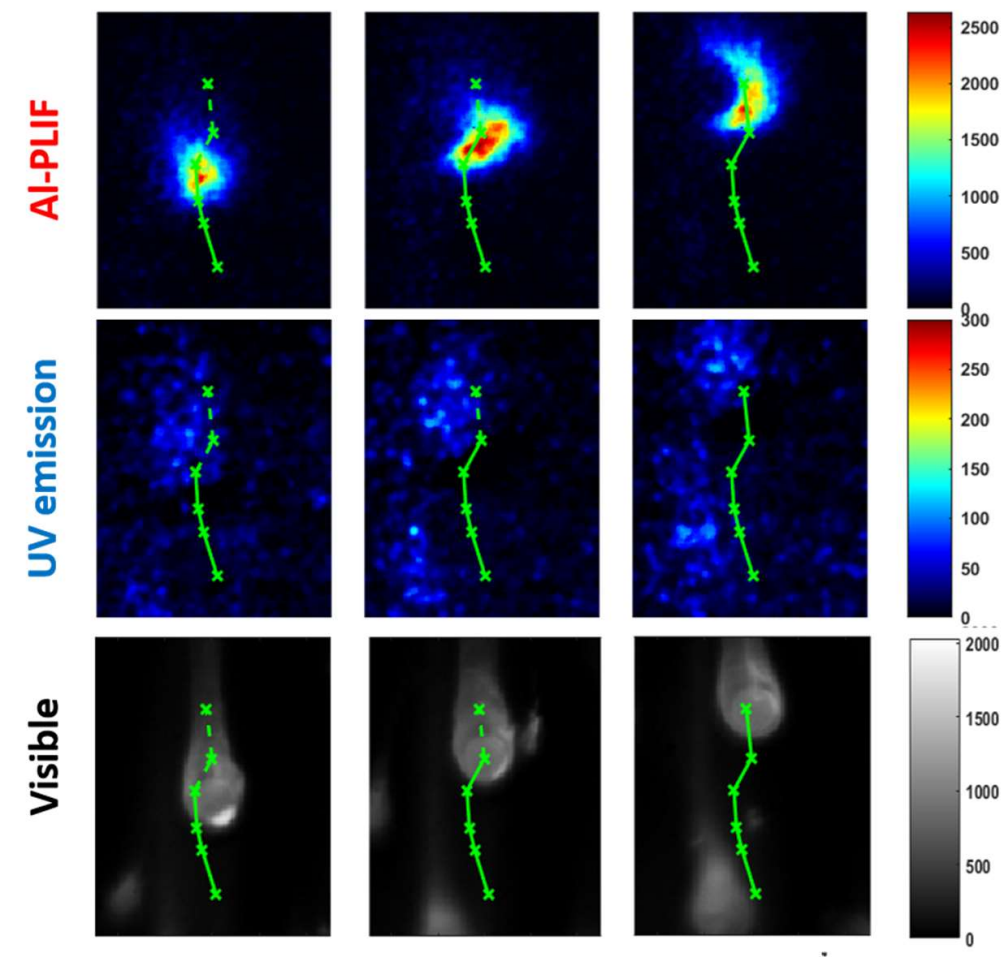

Figure 5: Tracked aluminum droplet images. The first row corresponds to the Al-PLIF images The second row shows UV-emission images (laser off) taken after each Al-PLIF images. The bottom row corresponds to the visible images taken simultaneously with the Al-PLIF images.

\section{B. Al-droplet combustion simulation}

Flame simulations are performed using the 1D combustion model presented in section IV.B. The chemical kinetic mechanism for surface reactions includes the reactions for Al evaporation and aluminum oxides from Ref. [6]. The gas-phase kinetic mechanism from Ref. [6] is extended by adding reactions for HCO and thermal NO from GRI-Mech v3.0.

The ambient gas conditions correspond to the equilibrium combustion products of the solid propellant without aluminum. This assumption is based on the following observations. The analyzed droplet is located at a distance from the regression surface, where the gas state becomes sufficiently homogeneous taking into account relatively small particle size in the composite propellant. The experimental images do not show any visible interference between the burning droplets.

Geometrical dimensions of the droplet and oxide cap are measured on the direct images. Mean values of these dimensions are used in the flame simulations.

Simulation results are obtained for 3 different positions of the outer boundary of the computational domain: $R_{e} / R_{p}=$ 2.0, 2.4, 3.0. The boundary radius $R_{e}$ is related to the droplet radius $R_{p}$. The obtained radial profiles of temperature and Al-atom concentration are shown in Figure 6. The temperature in the flame is normalized by the ambient temperature $T_{a}$, whereas the $\mathrm{Al}$ concentration is related to the value on the droplet surface [Al $]_{p}$. The flame temperature is strongly dependent on the $R_{e} / R_{p}$ parameter because the reaction rates are not high enough to compensate the change in heat and mass transfer across the outer boundary. The temperature maximum is located within a distance of $1.5 R_{p}$, which corresponds to the apparent flame radius on the direct images. The profiles of relative $\mathrm{Al}$ concentration demonstrate that about $90 \%$ of $\mathrm{Al}$ vapor is consumed within a distance of $1.1 R_{p}$. Both profiles are further used for simulating the Al-PLIF images presented in the following sub-section. 


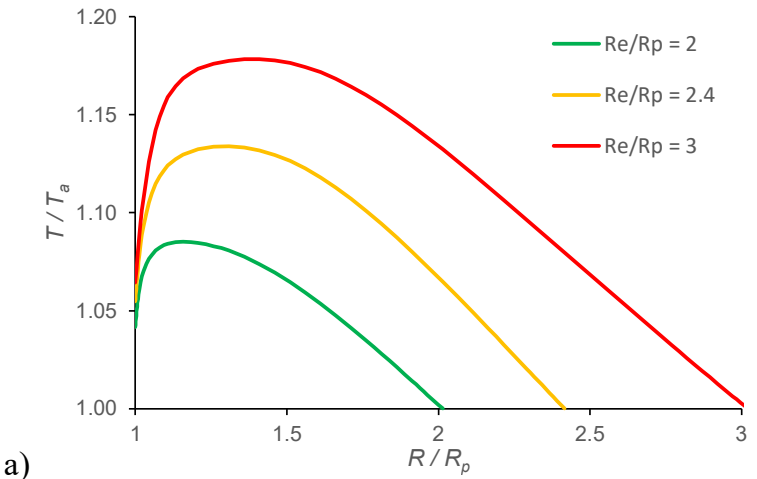

a)

Figure 6. Radial profiles of relative temperature and Al concentration in the flame for different positions of the outer boundary.

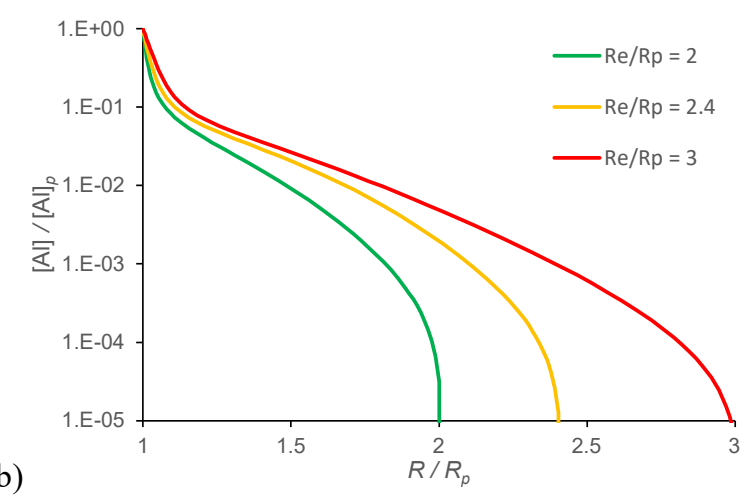

b)

\section{Simulated Al-PLIF images}

Al-PLIF simulation is described here for comparison with the third Al-PLIF image shown in Figure 5. The clear experimental quarter-moon crescent shape was chosen as target in order to illustrate Al-PLIF simulation trends.

\section{Laser attenuation}

Al-PLIF image simulation is performed using the model presented in section IV.B. The algorithm uses the temperature and Al-concentration levels from the combustion simulation shown in the previous sub-section. The LIF signal variations around the droplet are strongly dependent on Al concentration. The number density of Al atoms close to the droplet is large enough to totally absorb the laser sheet before it reaches the droplet surface. The lack of laser energy close to the droplet surface influences the LIF signal spatial distribution. Figure 7 shows lasertransmission levels for two laser-sheet positions, simulated for $\mathrm{Re} / \mathrm{Rp}=3.0$. Figure 7(a) corresponds to laser-sheet position $Z=0$. The laser sheets goes from right to left in the images. Laser intensity is fully transmitted as close to the droplet as $\mathrm{X}=2.4 \mathrm{Rp}$. Laser transmission is still evaluated at $80 \%$ for $\mathrm{X}=2 \mathrm{Rp}$. The laser is totally absorbed for $\mathrm{X}=1.7 \mathrm{Rp}$. The laser-transmission distribution shows a circle arc on the right-hand side of the droplet, which is reminiscent of the quarter-moon crescent shape visible in the experimental LIF images in Figure 5. Figure 7(b) shows the spatial laser transmission simulated around the droplet for laser sheet position $\mathrm{Z}=2 \mathrm{Rp}$. The aluminum droplet is behind the laser sheet from the camera point of view. Al concentration is reduced by a factor of 10000 in this region (Figure 6(b)) compared to the $Z=0$ area. Laser-sheet attenuation is then less intense with laser-excitation possible closer to the droplet position. 


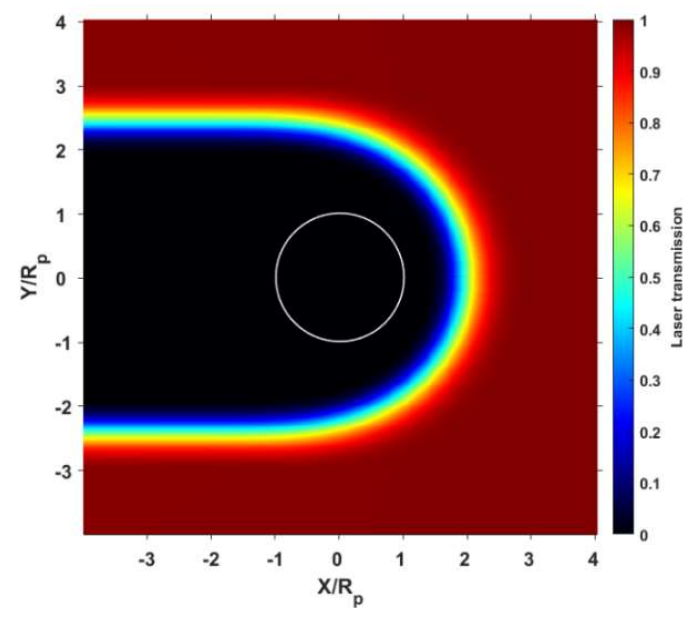

(a) $\mathrm{Z}=\mathbf{0}$

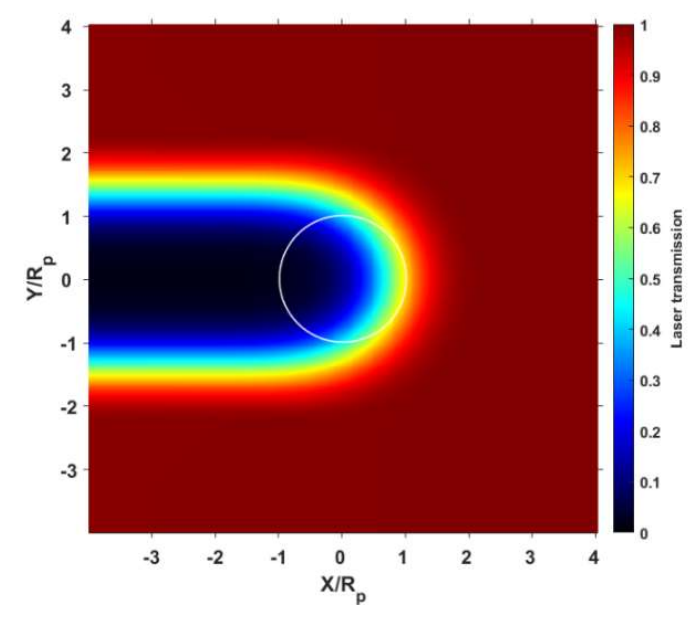

(b) $Z=2 R p$

Figure 7: Simulated laser-transmission levels for two laser-sheet positions. Simulation for $\operatorname{Re} / \mathbf{R p}=\mathbf{3 . 0}$.

\section{Effect of domain frontier position}

As shown in Figure 8, Al-PLIF images have been simulated for the three boundary-radius conditions presented in section V.B. Images in Figure 8 are all calculated for laser sheet position $Z=0$. The quarter-moon crescent shape is observed for every simulated case. For $\mathrm{Re}=2.0 \mathrm{Rp}$, the laser sheet reaches the droplet surface. For $\mathrm{Re}=2.4 \mathrm{Rp}$ and $\mathrm{Re}=3.0 \mathrm{Rp}$, the laser sheet is totally absorbed before reaching the droplet surface. The LIF-signal area extends further from the droplet with increasing $\mathrm{Rp}$, showing that $\mathrm{Al}$ atoms are present further from the droplet when simulation boundary is increased. Al-PLIF areas for $\mathrm{Re}=2.0 \mathrm{Rp}$ and $\mathrm{Re}=2.4 \mathrm{Rp}$ seem too narrow compared to the target experimental fluorescent region from Figure 5. In the rest of the article, only $\mathrm{Re}=3.0 \mathrm{Rp}$ is used for comparison with experimental images.
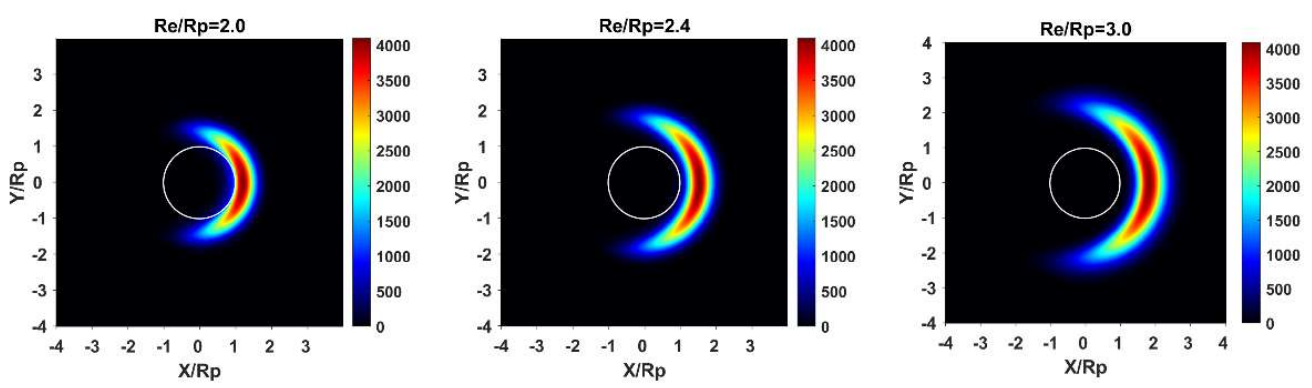

Figure 8: Simulated Al-PLIF images for three boundary radius $R e$ for laser-sheet position $Z=0$.

\section{Effect of laser-sheet position Z on signal morphology}

Simulations are performed for eight laser sheet positions relative to the droplet center, between $Z=-2.0 \mathrm{Rp}$ and $\mathrm{Z}=3.0 \mathrm{Rp}$. Simulated images are presented in Figure 9. Each image is normalized to its own signal maximum and adjusted to the camera dynamic range. This way, the impact of laser-sheet position on the shape of spatial distribution of signal is clearly visible. For $Z=-2 R p$, the signal spatial distribution is eclipsed by the droplet surface. This case has not been observed experimentally in any image. Signal morphology is similar for $Z=0 \mathrm{Rp}, 0.5 \mathrm{Rp}$ and $1.0 \mathrm{Rp}$ with thin crescent shapes. The signal left border gets closer to the droplet surface when laser-sheet distance to the droplet increases. For $Z=1.5 \mathrm{Rp}$ to $3.0 \mathrm{Rp}$, signal morphology goes from a quarter-moon crescent to a half-moon 
shape. The signal spatial distribution partially covers the droplet. Its height gradually decreases to be similar to the droplet diameter. The simulated images seem consistent to the three measured images shown in Figure 5.

In Figure 10, all eight Al-PLIF simulated images are normalized to a common value, i.e. to the maximum signal value obtained for $Z=0 \mathrm{Rp}$. Since signal intensity depends on laser sheet position, Figure 10 illustrates the predicted signal variation for a droplet moving along $Z$ relative to the laser sheet. Signal level becomes weaker than 1000 counts for $Z>2.5 \mathrm{Rp}$. Figure 11 presents horizontal profiles extracted from the simulated images from Figure 10. The peak signal is reduced by $10 \%$ at $Z=0.5 \mathrm{Rp}$, by $30 \%$ at $Z=1.0 \mathrm{Rp}$, by $50 \%$ for $Z=1.5 \mathrm{Rp}$ and by $90 \%$ at $Z$ $=3.0 \mathrm{Rp}$. The peak signal location also gets closer to the droplet center with increasing Z. Amplitude and shape of signal profile is strongly dependent on the droplet position relative the laser-sheet position.
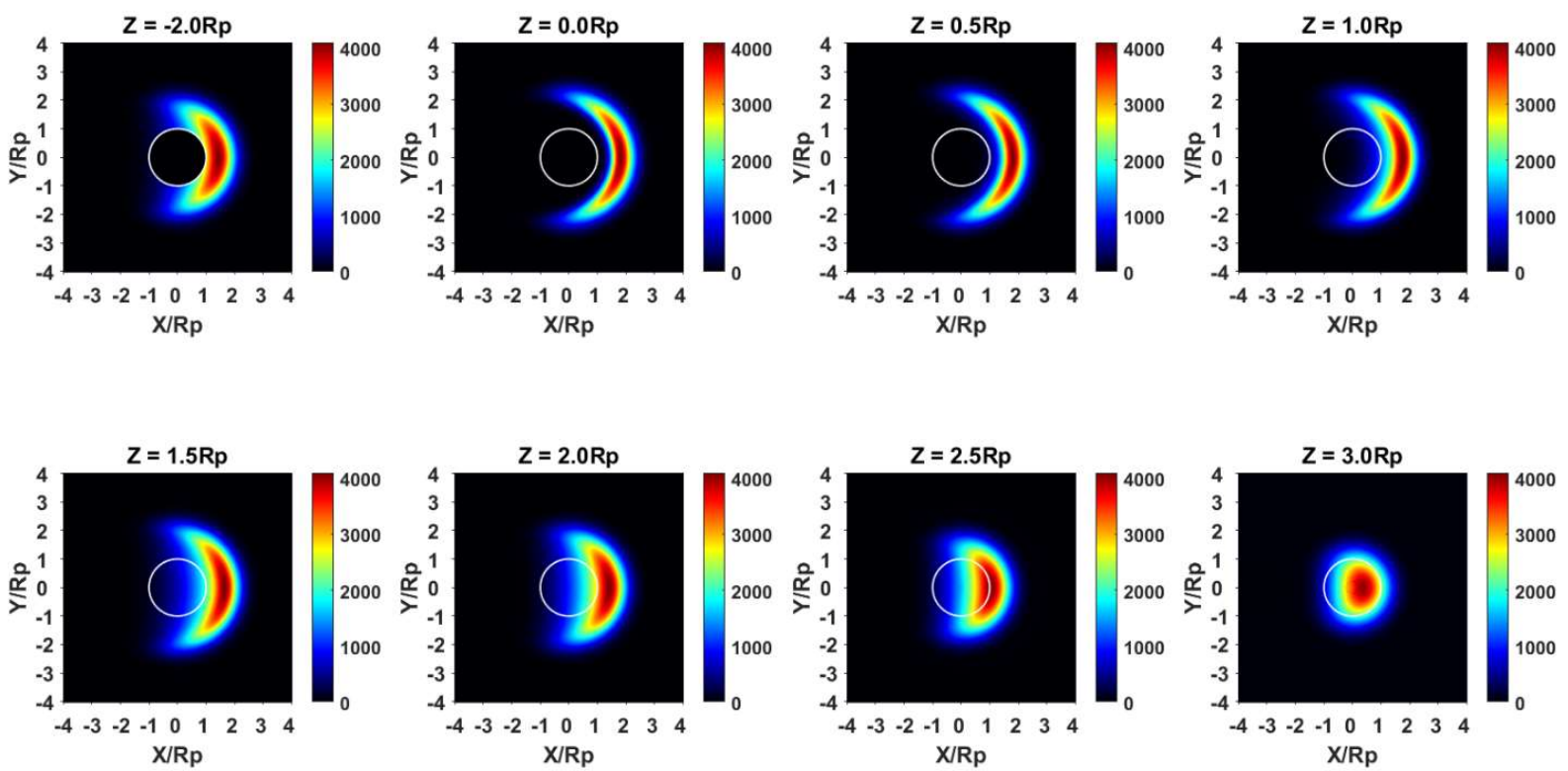

Figure 9: Al-PLIF images simulated for $R e=3 R p$ conditions. $L I F$ signal is normalized relative to their own maximum taking account for the camera dynamic. Simulation for laser sheet positions from $Z=-2 \operatorname{Rp}$ to 3

Rp. 


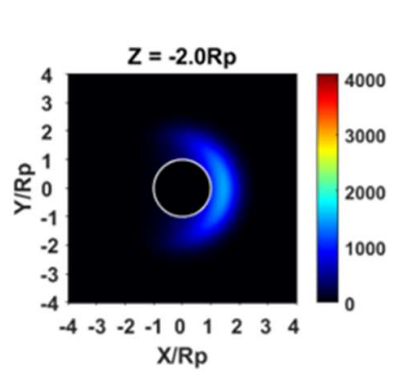

\section{Reference signal}
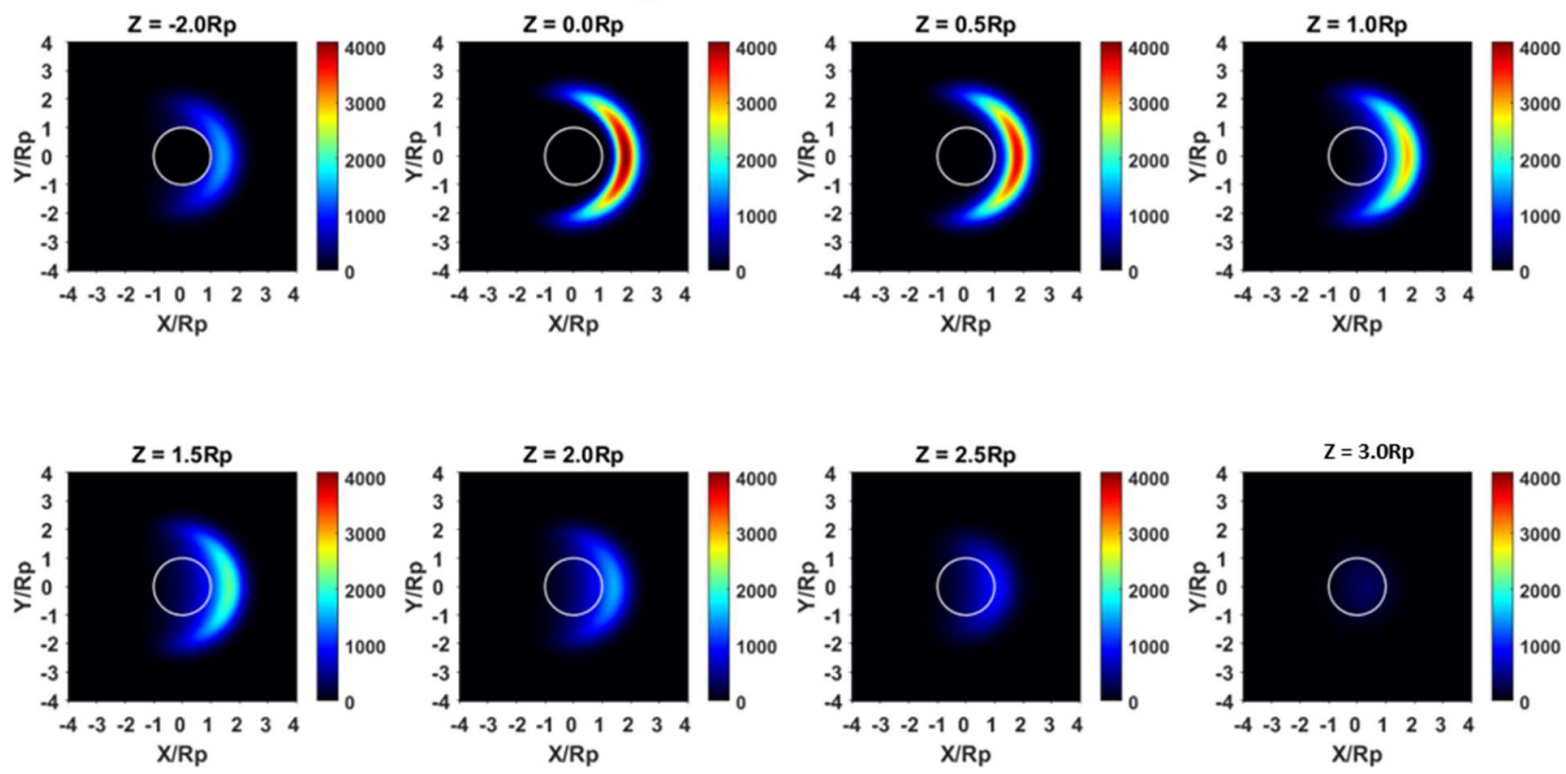

Figure 10: Al-PLIF images from Figure 11 with signal normalized to the maximum for laser sheet position $\mathbf{Z}=\mathbf{0}$.

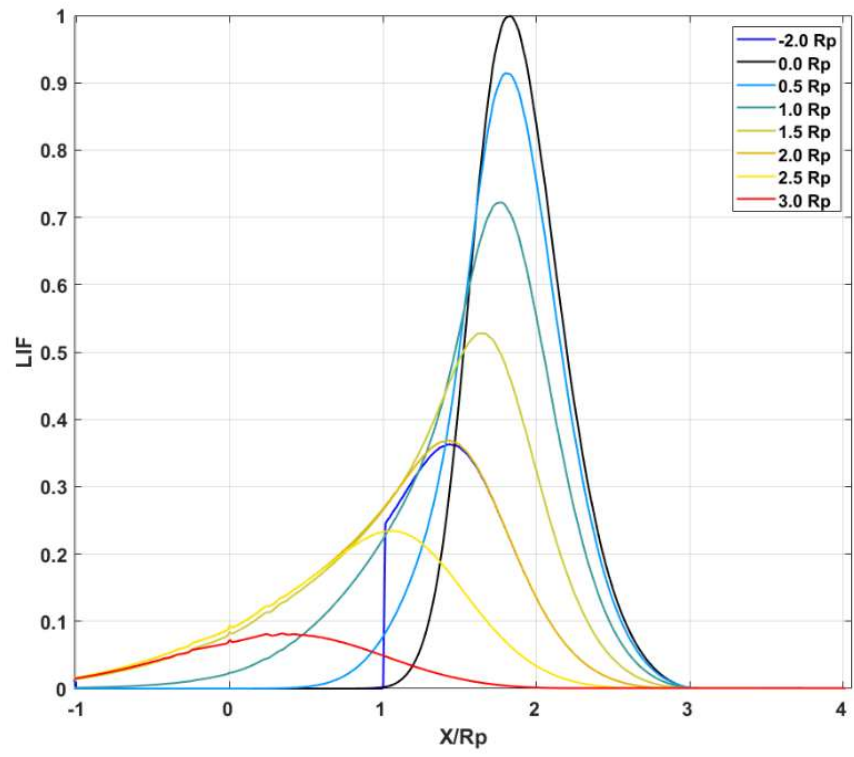

Figure 11: Horizontal profiles extracted from Al-PLIF simulated images presented in Figure 10. Simulation for $R e=3 R p$ conditions. Profiles for eight laser-sheet $Z$ positions.

D. Simulated Al-PLIF images compared to experimental images

\section{Sensitivity to relative droplet position on experimental LIF images}

Initial matching of the visible-emission and Al-PLIF images is done using an optical target during the camera calibration procedure. Precision of this method is estimated of a few pixels, which is a good result with respect to 
the overall image size. Nevertheless even a small displacement of the droplet center causes significant changes in the radial profiles of LIF signal. It is then decided to adjust the droplet position manually trying to match a pair of corresponding images regarding their topology. The sample images shown in Figure 12 are chosen for matching because the fluorescent zone has comparable dimensions with respect to the droplet. The droplet contour is first drawn manually on the direct image then it is placed on the PLIF image together with contours of constant luminosity to visualize the direct image topology. The irregular shape of the highly luminous zone on the PLIF image fits well the alumina cap edge on the lower side of the droplet.

For the PLIF image used for further analysis, matching is made by using the droplet displacement measured from the corresponding visible-emission images. The result of this procedure is shown in Figure 13. The overall shape of the fluorescent zone resembles to that in simulated images presented above, however signal intensity is relatively low in the upper half of the emission zone. Such an effect may be caused by the oxide cap located on the left upper side of the droplet according to the direct image.

In order to enable a more quantitative comparison to simulated signal, Al-LIF intensity profiles are extracted for various angles from the experimental image, presented in Figure 14. Profiles are only plotted for the lower quadrant, below the horizontal diameter, in order to reduce possible disturbance by the alumina cap. The visible image from Figure 13 suggests that the alumina cap is located on the right-hand side just above the horizontal diameter, which reduced $\mathrm{Al}$ evaporation from the droplet surface in this area. The evaporation reduction in this part might explain that signal level is slightly weaker in the upper half of the crescent shape from Figure 13 than in the lower part. The signal reduction is also visible in the profiles from Figure 14 with signal maxima for angle $0^{\circ}$ to $-30^{\circ}$ lower than for $-60^{\circ}$. The profiles also show that no LIF signal is obtained for distances larger than $3 \mathrm{Rp}$ from the droplet. 

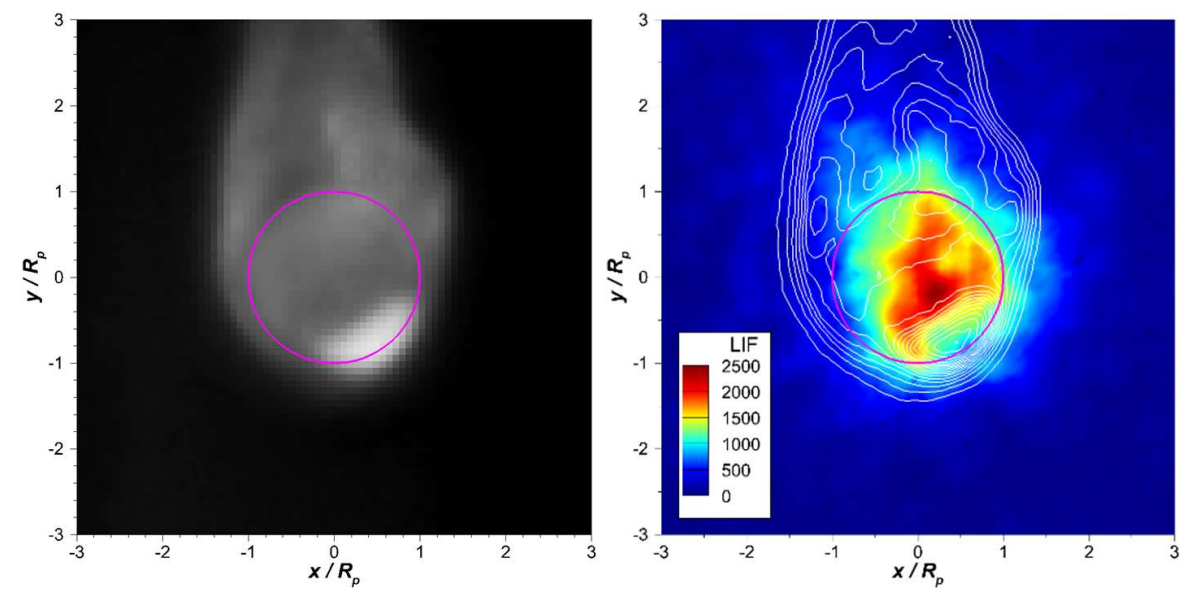

Figure 12. Visible-emission (left) and Al-PLIF (right) images used for adjustment. The magenta circles mark the doplet contour. Contours of constant luminosity from the direct image are superimposed on the PLIF image.
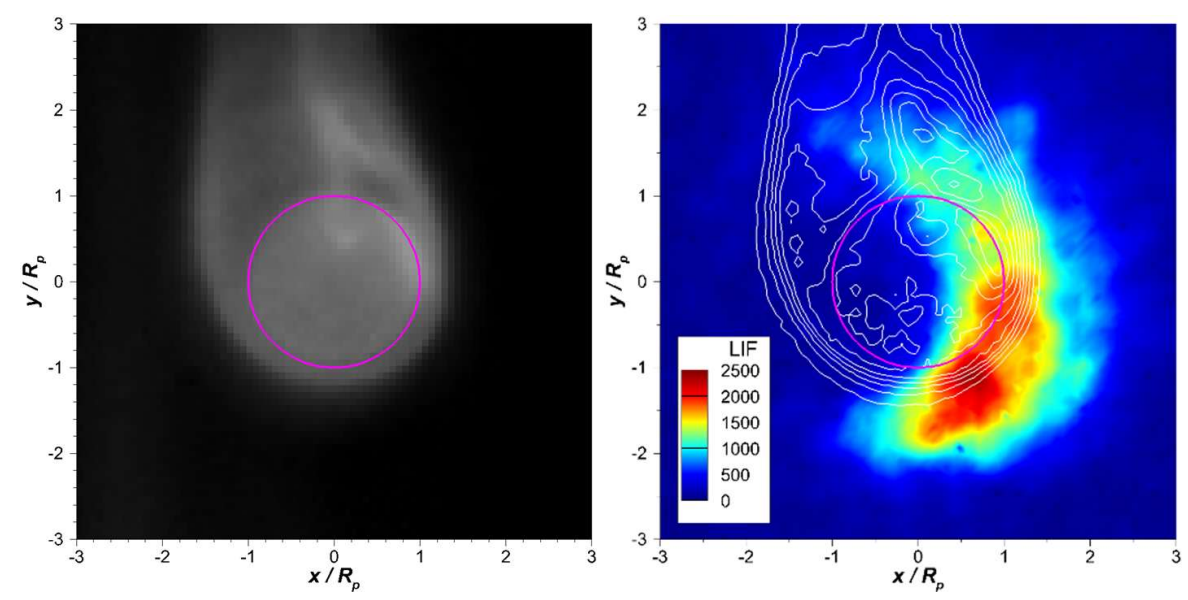

Figure 13. Visible-emission (left) and Al-PLIF (right) images used for analysis. The magenta circles mark the doplet contour. Contours of constant luminosity from the direct image are superimposed on the PLIF image. 


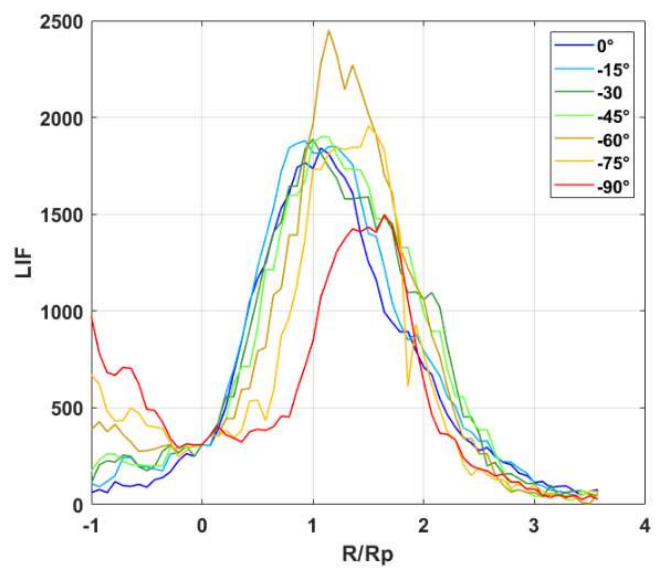

Figure 14. Al-PLIF intensity profiles extracted from Figure 13. Angles increase clockwise with $0^{\circ}$ for horizontal diameter. Negative values correspond to the bottom half relative to the diameter.

\section{Comparison between simulation and measurement for Al-PLIF}

Figure 15 reproduces the Al-PLIF experimental signal from Figure 13 with two simulated images. For both simulated images, signal level was adjusted to a $0-2500$ scale since 2500 is the maximum level from the experimental image. This shows reasonnable consistency between the overall crescent shapes and signal distribution within the shape, at least for the lower half of the images.
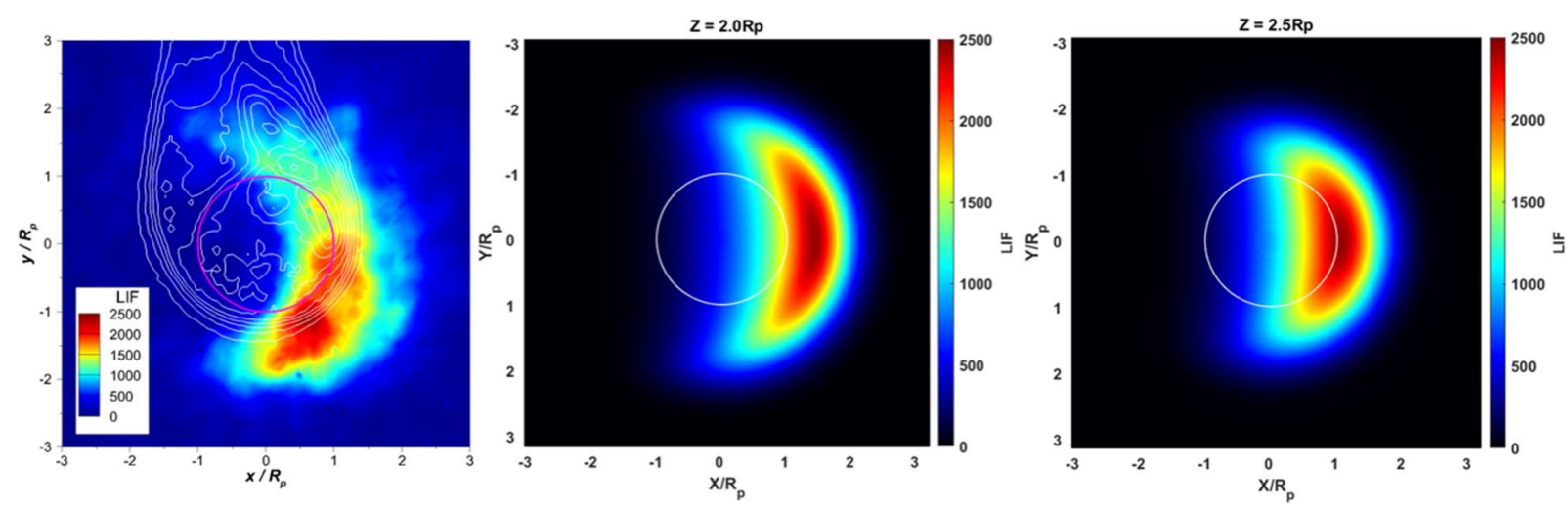

Figure 15. Experimental Al-PLIF image compared to simulated images for two laser-sheet positions $Z$. Simulation for $\operatorname{Re} / \operatorname{Rp}=\mathbf{3 . 0}$. 

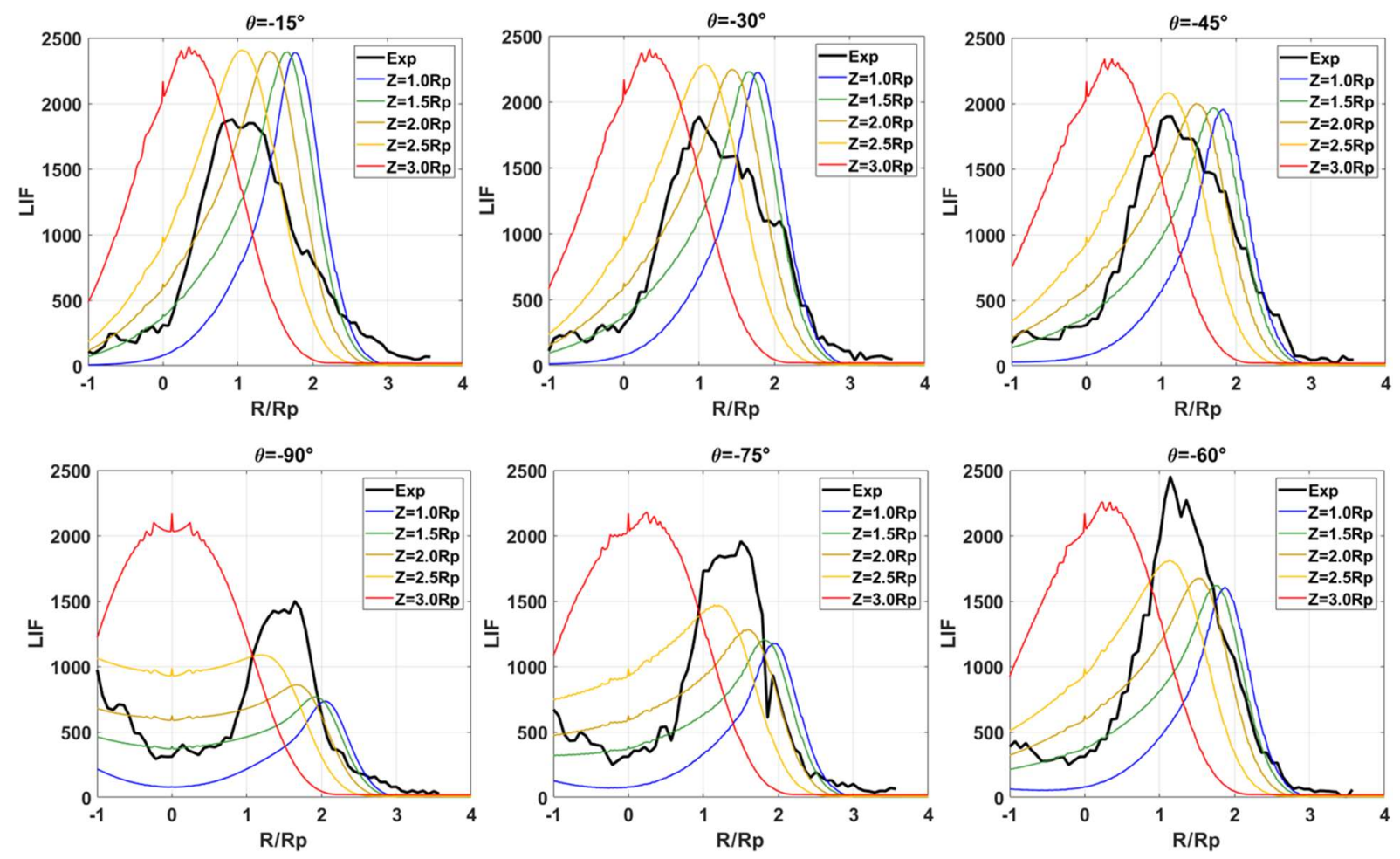

Figure 16: Comparison between experimental and simulated Al-LIF profiles for various laser-sheet positions $(Z)$ and various angles $(\theta)$.

Radial LIF profiles of signal are plotted in Figure 16 for both experimental and simulated data. Each plot correspond to profiles going through the droplet center at specific angles relative to the horizontal diameter, from $15^{\circ}$ to $-90^{\circ}$. Simulated data are plotted for five laser-sheet positions ragning from $Z=1.0 \mathrm{Rp}$ to $Z=3 \mathrm{Rp}$. It shows more quantitatively that the experimental data are rather consistent with laser-sheet positions $2.0 \mathrm{Rp}$ and $2.5 \mathrm{Rp}$ regarding peak position (peaks found for distances around 1.5 Rp) and LIF-area extension (up to 2.5 to $3.0 \mathrm{Rp}$ away from droplet center). Peak values do not match perfectly for each angle but trends are rather consistent with the angle: discrepancy between simulation for $Z=2.0 \mathrm{Rp}$ and measurements is no larger than $40 \%$. This is very promising because this is the very first confrontation of Al-PLIF simulated data to measurements, based only on a limited number of combustion simulation cases.

\section{VI.Conclusion}

A comparison approach between experimental and numerical Al-PLIF images has been presented to investigate aluminum-droplet combustion in solid-propellant flames. Al-PLIF images have been recorded within AP/HTPBbased solid-propellant flames at 1.0 MPa. An automatic detection and tracking algorithm method has been applied to on Al-PLIF images to provide signal evolution over time for various droplets above the solid propellant surface. One-dimensional quasi-steady aluminum droplet combustion simulations were performed for various external boundary positions. Simulations of LIF images have been performed by using Al-vapor concentration and temperature profiles from the combustion simulation. The laser sheet position $\mathrm{Z}$ affects both the amplitude and the shape of the spatial distribution of LIF signal. Al-LIF signal morphology goes from a quarter-moon crescent when the laser sheet is centered on the droplet to a half-moon shape when the laser sheet is further away from the droplet. Finally, Al-LIF profiles extracted from experimental and simulated images were compared. Simulated profiles trends are consistent with experimental profiles. This first comparison is very promising for the further comparisons between aluminum-droplet combustion simulations and Al-PLIF experimental data, enabling future improvements in aluminum-combustion models. 


\section{Acknowledgments}

We acknowledge CNES for its financial support to a large part of the present work.

\section{References}

[1] Gallier, S. and Godfroy, F, « Aluminum Combustion Driven Instabilities in Solid Rocket Motors ». Journal of Propulsion and Power, Vol. 25, No. 2,2009.

[2] Fabignon, Y., Anthoine, J., Davidenko, D., Devillers, R., Dupays, J., Gueyffier, D., and Erades, C. « Recent advances in research on solid rocket propulsion », AerospaceLab, (11),2016,pp. 15.

[3] Genot, A., Gallier, S., and Schuller, T. "Thermo-acoustic instabilities driven by fuel droplet lifetime oscillations ». Proceedings of the Combustion Institute, 37(4), 2019, pp. 5359-5366.

[4] Washburn, E. B., Webb, J. A., and Beckstead, M. W., « The simulation of the combustion of micrometer-sized aluminum particles with oxygen and carbon dioxide ». Combustion and flame, 157(3), 2010, pp. 540-545.

[5] Catoire, L., Legendre, J. F., and Giraud, M., « Kinetic model for aluminum-sensitized ram accelerator combustion ». Journal of propulsion and power, 19(2), 2003, pp.196-202.

[6] Glorian, J., Gallier, S. and Catoire, L., « On the role of heterogeneous reactions in aluminum combustion », Combustion and Flame, Vol. 168, 2016, pp. 378-392.

[7] Bucher, P., Yetter, R. A., Dryer, F. L., Parr, T. P., Hanson-Parr, D. M., and Viceni, E. P., "Flames structure measurement of single, isolated aluminum particles burning in air. In Symposium (International) on Combustion, Vol. 26, No. 2,1996, pp. 1899-1908.

[8] Bucher, P., Yetter, R. A., Dryer, F. L., Parr, T. P., and Hanson-Parr, D. M., «PLIF species and ratiometric temperature measurements of aluminum particle combustion in $\mathrm{O} 2, \mathrm{CO} 2$ and $\mathrm{N} 2 \mathrm{O}$ oxidizers, and comparison with model calculations", In Symposium (International) on Combustion, 1998, Vol. 27, No. 2, pp. 2421-2429.

[9] Braconnier, A., Gallier, S., Halter, F., and Chauveau, C., « Aluminum combustion in CO2-CO-N2 mixtures », Proceedings of the Combustion Institute, 2021,38(3),pp. 4355-4363.

[10] Chen, Y., Guildenbecher, D. R., Hoffmeister, K. N., Cooper, M. A., Stauffacher, H. L., Oliver, M. S. ,and Washburn, E. B., "Study of aluminum particle combustion in solid propellant plumes using digital in-line holography and imaging pyrometry. », Combustion and Flame, 2017, 182, pp. 225-237.

[11] Vilmart, G., Dorval, N., Orain, M., Lambert, D., Devillers, R., Fabignon, Y., and Bresson, A. « Detection of iron atoms by emission spectroscopy and laser-induced fluorescence in solid propellant flames. », Applied optics, 2018, 57(14),pp. 3817-3828.

[12] Beckstead, M. W., Liang, Y., and Pudduppakkam, K. V. « Numerical simulation of single aluminum particle combustion. » Combustion, Explosion and Shock Waves, 41(6), 2005, pp. 622-638.

[13] Washburn, E. B., Trivedi, J. N., Catoire, L., and Beckstead, M. W., « The simulation of the combustion of micrometer-sized aluminum particles with steam. », Combustion Science and Technology, 180(8), 2008, pp. $1502-1517$.

[14] Vilmart, G., Dorval, N., Devillers, R., Fabignon, Y., Attal-Trétout, B., and Bresson, A. , « Imaging Aluminum Particles in Solid-Propellant Flames Using 5 kHz LIF of Al Atoms. », Materials, 12(15), 2019, pp. 2421.

[15] Chevalier, P. H., Devillers, R., Dorval, N., Vilmart, G., Attal-Trétout, B., Brossard, C., and Pichillou, J. , " Analysis of high-speed Al-PLIF images to study aluminum droplet combustion in solid propellant flames. » In EUCASS $2019,2019$.

[16] Vilmart, G., Dorval, N., Attal-Tretout, B., and Bresson, A. « Detection of iron and aluminum atomic vapors by LIF technique: application to solid propellant combustion. »In 33rd AIAA Aerodynamic Measurement Technology and Ground Testing Conference, 2017, pp. 3900. 
[17] Nugue, M., Devillers, R. W., Le Besnerais, G. and Cesco, N. , « Particle detection \& size evaluation in solid propellant flames via experimental image analysis to improve two-phase flow simulation in rocket motors. » Space Propulsion conference, 2016.

[18] Devillers, R.W., Dorval, N., Vilmart, G., Nugue, M., Le Besnerais, G. and Pichillou, J., « Aluminum particle tracking on experimental shadowgraphy and al-plif images to provide velocity data for two-phase flow simulations of solid rocket motors », Space Propulsion 2018, 2018.

[19] Matas, J., Chum, O., Urban, M. and Pajdla, T. , «Robust Wide Baseline Stereo from Maximally Stable Extremal Regions », Proc. Of British Machine Vision Conference, 2002, pp. 384-396

[20]Muller, M., Davidenko, D., and Giovangigli, V., « Computational study of aluminum droplet combustion in different atmospheres », $7^{\text {th }}$ European Conference for Aeronautics and Space Sciences (EUCASS 2017), 2017

[21]Muller, M., Davidenko, D., and Giovangigli, V. 2019. «Computational study of Al droplet combustion in a burning solid propellant environment », $8^{\text {th }}$ European Conference for Aeronautics and Space Sciences (EUCASS 2019), 2019.

[22]Ern, A., and Giovangigli, V., « Fast and accurate multicomponent transport property evaluation », Journal of Computational Physics, Vol. 120,1995, pp. 105-116.

[23]Daily, J.W., "Laser induced fluorescence spectroscopy in flames », Prog. Energy Combust. Sci. 23, 1997,pp. 133-199.

[24] Vilmart, G., « Détection de vapeurs d'atomes métalliques par fluorescence induite par laser (LIF) : application à la propulsion solide », $\mathrm{PhD}$ Thesis,2017

[25] C. T. J. Alkemade, T. Hollander, W. Snelleman, and P. J. T. Zeegers, «Metal Vapours in Flames, 2017

[26]Johnson, M. R., Devillers, R. W., and Thomson, K. A., «A generalized Sky-LOSA method to quantify soot/black carbon emission rates in atmospheric plumes of gas flares ». Aerosol Science and Technology, 47(9), 2013 ,pp. 1017-1029.

[27] “Atomic spectral line database from CD-ROM 23 of R. L. Kurucz." Available: https://www.cfa.harvard.edu/amp/ampdata/kurucz23/sekur.html. 